\title{
Temperature Anisotropies and Distortions Induced by Hot Intracluster Gas on the Cosmic Microwave Background.
}

\author{
F. Atrio-Barandela ${ }^{1}$ \\ Física Teórica. Universidad de Salamanca. 37008 Spain. \\ email: atrio@astro.usal.es \\ J. P. Mücket \\ Astrophysikalisches Institut Potsdam. D-14482 Potsdam. \\ email: jpmuecket@aip.de
}

\begin{abstract}
The power spectrum of temperature anisotropies induced by hot intracluster gas on the cosmic background radiation is calculated. For low multipoles it remains constant while at multipoles above $l>2000$ it is exponentially damped. The shape of the radiation power spectrum is almost independent of the average intracluster gas density profile, gas evolution history or clusters core radii; but the amplitude depends strongly on those parameters. Its exact value depends on the global properties of the cluster population and the evolution of the intracluster gas. The distortion on the Cosmic Microwave Background blackbody spectra varies in a similar manner. The ratio of the temperature anisotropy to the mean Comptonization parameters is shown to be almost independent of the parameters of the cluster model and, in first approximation, depends only on the number density of clusters. An independent determination of the contribution of clusters to the distortion of the blackbody spectrum and the temperature fluctuations of the Cosmic Microwave Background would determine the number density of clusters that contribute to the Sunyaev-Zel'dovich effect.
\end{abstract}

Subject headings: Cosmic Microwave Background. Cosmology:theory. Galaxies:clusters:general. 


\section{Introduction.}

The Compton scattering of the Cosmic Microwave Background (CMB) photons by the hot gas present in clusters of galaxies, was first described by Sunyaev \& Zel'dovich (1970). The Sunyaev-Zel'dovich effect -hereafter SZ- has two components: thermal and kinematic. The first is caused by the random thermal motions of the electrons whose distribution is assumed to be isotropic (in the cluster reference frame). The kinematic component is an additional effect due to the peculiar velocity of the cluster with respect to the CMB (see Rephaeli 1995 for a review). Nowadays, several groups are searching or are setting up telescopes to measure the SZ effect on single clusters (Holzapfel et al. 1997, Jones 1996, Saunders 1996). But besides the effect of individual clusters the entire cluster population acts as a screen of scattering sites through which the background photons must pass to reach the observer. The overall effect is both to distort the black body spectrum and to induce temperature anisotropies on the CMB. The latter is an important component of the full anisotropy on angular scales of several arcminutes (Cole \& Kaiser 1989, Bartlett \& Silk 1994, Colafrancesco et al. 1994). The anisotropy depends on the cluster richness, its evolution and on the cosmological model. Therefore, detection of anisotropies and distortions will yield significant insight into the cosmological evolution of clusters.

The analysis of the COBE/FIRAS data yielded an upper limit on the degree of Comptonization of the CMB (determined by the parameter $\bar{y}$ to be defined below) of $\bar{y} \leq 1.5 \times 10^{-5}$ at $95 \%$ confidence level (Mather et al. 1994). The Planck satellite, scheduled to be launched in the next decade, will not specifically look for distortions. Those will be deduced from the data on temperature anisotropies. At the present stage several groups are studying methods to substract foregrounds (Tegmark \& Efstathiou 1996, Hobson et al. 1998) and are performing simulations to predict the sensitivity level at which cosmological parameters can be measured. In this respect, it is necessary to carry out a careful analysis of the power spectrum of the foregrounds since this will allow to distinguish at each frequency between the genuine CMB distortions and the contribution of clusters and other foregrounds. As remarked by Tegmark \& Efstathiou (1996) this improves the technique of removing foregrounds simply by comparing data at different frequencies.

In this paper we shall obtain the radiation power spectrum of CMB temperature anisotropies induced by the entire population of clusters of galaxies, the mean distortion and the ratio of the temperature anisotropy measured by SuZie (Church et al. 1997) to the Comptonization parameter. We will show that distortions and temperature anisotropies are related not only for a single cluster but also for the values averaged over all clusters. We extend the work of Bartlett \& Silk (1994) and Colafrancesco et al. (1997) by computing the power spectrum and analyzing its dependence with the parameters describing the cluster population and the number density. Contrary to the latter authors, we do not make a detailed analysis of all cosmological models. We shall restrict our study to the standard Cold Dark Matter model.

The outline of the paper is as follows: in Sec. 2 , we rederive the power spectrum of the temperature anisotropies due to the contribution of clusters of galaxies. In Sec. 3, we integrate our equations assuming a number density of clusters given by the Press-Schechter formalism. We give the shape of the power spectrum and its dependence on properties of the average SZ cluster population. In Sec. 4, we show that while the amplitude of the radiation power spectrum and the CMB distortion varies strongly with the main cluster parameters, the ratio of the temperature fluctuation to the mean Comptonization parameter is almost independent of the parameters of the cluster model. In first approximation, this ratio is determined solely by the number density of clusters. Finally, in Sec. 5, we present our main conclusions.

\section{Temperaure anisotropies induced by clus- ters of galaxies.}

The passage of CMB photons through a single cluster of galaxies distorts the radiation spectrum due to the inverse Compton scattering. The amplitude of the distortion depends on frequency. It can be described more appropriately in terms of the change in brightness on the CMB. The temperature difference is given by

$$
\frac{\delta T}{T_{o}}=g(x) y_{c},
$$

where $x=h \nu / k T$ is the CMB frequency in dimensionless units and $g(x)=(x \operatorname{coth}(x / 2)-4)$ gives the frequency dependence of the effect. The cluster Comp- 
tonization parameter is defined as

$$
y_{c}=\frac{\sigma_{T} k_{B}}{m_{e} c^{2}} \int T n d l
$$

where $n$ and $T$ are the intracluster electron density and temperature, $\sigma_{T}$ is the Thomson cross section, $k_{B}$ is the Boltzmann constant, $m_{e}$ is the electron mass and the integral is performed along the line of sight through the cluster.

Clusters are known to be extended X-ray sources with gas density profiles well fitted by

$$
n_{e}(r)=n_{c}\left(1+\left(r / r_{c}\right)^{2}\right)^{-3 \beta / 2},
$$

where $n_{c}$ is the central electron density and $r_{c}$ is the core radius of the cluster. The observed values of $\beta$, obtained from X-ray surface brightness profiles, range from 0.5 to 0.7 (Jones \& Forman 1984, Markevitch et al. 1997). Throughout this paper we will adopt $\beta=2 / 3$ since it permits a more simplified treatment.

The distortion induced by the IC gas depends on the temperature profile along the line of sight through the cluster. While most authors assumed the IC gas to be isothermal, in a recent study encompassing 26 nearby ASCA clusters Markevitch et al. (1997) have shown that the gas temperature within clusters decreases slowly with radius. Note that the uncertainty about the ASCA point spread function implies that errors on the density profile of individual clusters are correlated. Therefore, their data can not be used to estimate the average properties of the IC gas. To simplify, we shall retain the hypothesis of isothermality since the computation becomes simpler, but keeping in mind that our analysis could be easily modified to take into account any dependence of temperature with radius.

If the virial radius of the cluster $r_{v}$ is expressed in terms of the core radius as $r_{v}=p r_{c}$, eq. [2] can be recast in the following manner:

$$
y_{c}=y_{o} \phi(\theta)
$$

with $y_{o}=\left[k_{B} \sigma_{T} / m_{e} c^{2}\right] r_{c} T n_{c}, \theta$ the angular separation between the line of sight and the center of the cluster and

$$
\phi(\theta)=\frac{2}{\sqrt{1+\left(\theta / \theta_{c}\right)^{2}}} \tan ^{-1}\left[\sqrt{\frac{p^{2}-\left(\theta / \theta_{c}\right)^{2}}{1+\left(\theta / \theta_{c}\right)^{2}}}\right] .
$$

In this last expression, $\theta_{c}$ is the angle subtended by the core radius of the cluster. Eq. 4 gives the effect produced by a single cluster through a particular line of sight. Note that $p$, given as the ratio between the virial and the core radius, is not a parameter but a function of mass $M$ and redshift $z$.

\subsection{Mean Comptonization Parameter.}

The mean distortion on the CMB induced by all clusters is obtained adding the effect of one cluster for all possible lines of sight:

$$
\bar{y}=\int \frac{d n}{d M} d M \frac{d V}{d z} d z \kappa y_{o} \bar{\phi}
$$

In this expression, $d n / d M$ is the cluster number density per unit of mass, $\kappa$ gives the probability that a particular line of sight crosses a cluster. This probability is simply $\kappa=\left(p \theta_{c}\right)^{2} / 4$. Finally, $\bar{\phi}$ is the averaged line of sight through a cluster:

$$
\bar{\phi}=\frac{\int \phi \theta d \theta}{\int \theta d \theta}=4 p^{-2}\left(p-\tan ^{-1} p\right) .
$$

It is useful to introduce the following notation:

$$
<\phi>\equiv \frac{1}{2} \int \theta d \theta \phi \text {. }
$$

This allows to write $\kappa \bar{\phi}=\langle\phi\rangle$. Now eq. [6] can be rewritten as follows:

$$
\bar{y}=\int \frac{d n}{d M} d M \frac{d V}{d z} d z y_{o}<\phi>.
$$

Notice that $\bar{y}$ can now be understood as the average of $y_{o}\langle\phi\rangle$ over the entire cluster population. The superposed effect is determined by the cosmological model, cluster abundance and redshift evolution of the IC medium (Colafrancesco et al. 1997).

\subsection{Radiation Power Spectrum.}

The IC gas does not only produce distortions on the CMB spectrum. It also induces temperature anisotropies. The contribution of a single cluster to the temperature anisotropy on a wavenumber $\mathbf{l}$ is

$$
\frac{\delta T}{T}(\mathbf{l})=\frac{1}{2 \pi} g(x) y_{o} \int d^{2} \theta \phi(\theta) e^{-i \mathbf{l} \boldsymbol{\theta}} .
$$

The power spectrum can be obtained by adding in quadrature the contribution of all clusters. Neglecting cluster spatial correlations, we can write (Cole \& Kaiser 1988; cp. also Bartlett \& Silk 1994)

$$
P(l)=\int \frac{d n}{d M} d M \frac{d V}{d z} d z\left(g(x) y_{o}\right)^{2}|\tilde{\phi}(l)|^{2},
$$


where $\tilde{\phi}(l)$ is the Fourier transform of the angular profile of the cluster. For scales much larger than the virial radius of a typical cluster, radiation temperature anisotropies originated by the contribution of Poisson distributed clusters lead to $P(l) \simeq$ const.

For scales $l>\theta_{c}^{-1}$ the behaviour of $P(l)$ can be obtained analytically taking the limit $p$ going to infinity (but keeping $p \theta_{c}$ finite). In this limit, the radiation power spectrum becomes exponentially damped, that is, it decreases faster than $l^{4}$ as expected by Tegmark \& Efstathiou (1996). In agreement with these authors, we shall demostrate numerically that $l^{2} P(l)$ has a coherence scale, i.e. a maximun, around $l \simeq 1000-2000$. For reference, let us mention that the angular scale $l=2000$ corresponds to a wavenumber $k \simeq 0.5 h \mathrm{Mpc}^{-1}$ or scalelength $\lambda \simeq 10 h^{-1} \mathrm{Mpc}$.

From the power spectrum we can compute the correlation function of temperature anisotropies. In the flat sky approximation, it is given by (AtrioBarandela, Gottlöber \& Mücket 1997)

$$
C(\alpha)=\frac{1}{2 \pi} \int l d l W(l) P(l) J_{o}(l \alpha),
$$

where $\mathrm{W}(\mathrm{l})$ represents the window function of the experiment. This expression can be rewritten in a more illuminating form. Replacing the power spectrum (eq. [11]), and after some algebra, for an experiment with infinite resolution and full sky coverage, we obtain:

$$
C(0)=\frac{1}{\pi} g(x)^{2} \int \frac{d n}{d M} d M \frac{d V}{d z} d z y_{o}^{2}<\phi^{2}>,
$$

where $\phi$ is given by eq. 5 and the average was defined in eq. [8]. This expression states that the correlation function at the origin represents the average of $y_{o}^{2}<\phi^{2}>$ over all clusters. For a single cluster, Compton parameter $y_{c}$ and temperature anisotropies are related (see eq. [1]). Comparison with eq. [9] suggest that a similar relation holds between $\sqrt{C(0)}$ and $\bar{y}$. In Sec. 4 we shall show that this is indeed the case. We shall particularize this relation for the SuZie experiment (Church et al. 1997) and with the upper limits obtained from their observations we shall set a constraint on $\bar{y}$ stronger than the one derived from COBE/FIRAS.

\section{Cluster Model.}

Numerical estimates of the mean Comptonization parameter, the power spectrum and the correlation function for a given experiment require the cluster number density and average properties, as a function of mass, redshift evolution of the IC gas and cosmological model, to be specified. The number density of clusters per unit of redshift is given by the PressSchechter formula (Press \& Schechter, 1974):

$$
\frac{d n}{d M}=\sqrt{\frac{2}{\pi}} \frac{\rho_{b}}{M} \frac{\delta_{v} b}{\sigma^{2}} \frac{d \sigma}{d M} \exp \left(-\frac{\delta_{v}^{2} b^{2}}{2 \sigma^{2}}\right),
$$

where $\rho_{b}$ is the background density at redshift $z, M$ is the virial mass of the cluster, $\sigma$ is the rms of the linear density fluctuation field at $z$, smoothed over the region containing $M, b$ is the bias factor and $\delta_{v}$ is the linear density contrast of a perturbation that virializes at $z$. The background density and the variance of the density field scale with redshift: $\rho_{b} \sim(1+z)^{3}, \sigma \sim(1+z)^{-1}$ while the bias and $\delta_{v}$ are assumed to be constant.

We shall restrict our analysis to a Cold Dark Matter model with Hubble constant $h=0.5\left(H_{o}=100 h\right.$ $\mathrm{km} \mathrm{s}{ }^{-1} \mathrm{Mpc}^{-1}$ ) and normalized to produce a rms matter density perturbation at a $8 h^{-1} \mathrm{Mpc}$ scale of $\sigma_{8}=0.7$. Only for this particular model and using the largest numerical simulation to date Tozzi \& Governato (1997) checked that for clusters with masses larger than $10^{14} \mathrm{M}_{\odot}$, the Press-Schechter formula was in reasonable agreement with the number density of clusters up to redshift $z=1$ if $\delta_{v} b=2.1$. A slightly larger value $\left(\delta_{v} b=2.6\right)$ was found by Colafrancesco \& Vittorio (1994) by fitting the standard CDM model to the observed cluster X-ray luminosity function. The results presented in the next section were obtained assuming the former value at all redshifts.

To compute the temperature anisotropies induced by hot IC gas, we need to translate the properties of a sample of clusters at low redshifts into their equivalents at earlier epochs. In what follows, the virial mass $M$ will be expressed in units of $10^{15} \mathrm{M}_{\odot}$. For the spherical collapse model, the virial radius scales as $r_{v}=r_{v o} M^{1 / 3}(1+z)^{-1}$ where $r_{v o}$ is the current average virial radius of a $10^{15} \mathrm{M}_{\odot}$ cluster. We shall use the entropy-driven model of cluster evolution developed by Bower (1997) to describe the core radius evolution as a function of redshift and mass. This model is applicable to an isothermal IC gas distribution. If temperature is proportional to the velocity dispersion of the dark matter then

$$
T=T_{g} M^{2 / 3}(1+z),
$$

where $T_{g}$ is a normalization constant. It corresponds 
to the current temperature of the IC gas of a cluster of $10^{15} \mathrm{M}_{\odot}$. In our numerical estimates we took $T_{g}=$ $10^{8} \mathrm{~K}$. The core density evolves as

$$
\rho_{c} \propto T^{3 / 2}(1+z)^{-3 \epsilon / 2},
$$

where $\epsilon$ parametrizes the rate of core entropy evolution. Therefore, the central electron density scales as: $n_{c}=n_{c o}\left(T / T_{g}\right)^{3 / 2}(1+z)^{-3 \epsilon / 2}$. From their study on the Luminosity - Temperature relation on clusters at high redshift, Mushotzky and Scharf (1997) found $\epsilon=0 \pm 0.9$.

For a cluster with $\beta=2 / 3$, assuming that $r_{v} \gg r_{c}$, eq. 16] leads to the following expression for the core radius:

$$
r_{c}=r_{c o} M^{-1 / 6}(1+z)^{(-1+3 \epsilon) / 4},
$$

where $r_{c o}$ is the average core radius today for a cluster of $10^{15} \mathrm{M}_{\odot}$. From this expression one obtains $p=$ $p_{o} M^{1 / 2}(1+z)^{-\frac{3}{4}(1+\epsilon)}$. In our numerical estimates, we took $r_{v o}=1.3 \mathrm{~h}^{-1} \mathrm{Mpc}$. The core radius today is given by $r_{c o}=r_{v o} / p_{o}$. In our analysis we have considered the effect of different core radii, i.e., $p_{o}=7,10,15$, since, according to Makino, Sasaki \& Suto (1997), the core radius of clusters is uncertain and could have been overestimated. Finally, we took the present gas density of a $10^{15} \mathrm{M}_{\odot}$ cluster to be $n_{c o}=2 \cdot 10^{-3} \mathrm{~cm}^{-3}$ (Peebles, 1993) for a Hubble constant of $h=0.5$.

We would like to remark that all the relations introduced in this section are to be thought accurate in determining the mean evolution of the cluster population as a whole. They should not be considered applicable to individual clusters.

\section{Results and Discussion.}

We obtained the power spectrum of temperature anisotropies induced by clusters of galaxies by numerically integrating eq. 11]. The main limitation of our work is to determine the scale of the less massive objects containing enough hot gas to produce a measurable effect on the CMB. We shall ellaborate on this point further below. Except otherwise specified, we assumed a lower limit $M_{l}=10^{14} \mathrm{M}_{\odot}$ since above that mass scale our cluster model is an adequate description of clusters in the local Universe (Mushotzky \& Scharf, 1997) and the Press-Schechter formula has been found to be accurate (Tozzi \& Governato, 1997). As an upper limit we took $M_{u}=2 \cdot 10^{15} \mathrm{M}_{\odot}$.

In Fig. (11) we plot the mean CMB temperature offset $T_{o}\left(l^{2} P(l) / 2 \pi\right)^{1 / 2}$. The CMB blackbody tempera- ture $T_{o}$ is expressed in units of $\mu \mathrm{K}$. We took $g(x)=1$ in all our plots, so the y-axis should be multiplied by the correct value when comparing with a particular experiment. One should notice that the mean temperature offset scales as: $T_{g} r_{c o} n_{c o} g(x)$ where the values of all these constants, given in the previous section, correspond to a $10^{15} \mathrm{M}_{\odot}$ cluster. In this way, the yaxis on Fig. (11) can be easily rescaled to a different set of values. All plots correspond to $\operatorname{CDM}\left(\Omega_{B}=0.05\right.$, $H_{o}=50 \mathrm{~km} \mathrm{~s}^{-1} \mathrm{Mpc}^{-1}$ ) normalized $\sigma_{8}=0.7$. In Fig. (11a) we consider the effect of different IC gas evolution histories: $\epsilon=-1$ (thin solid line), $\epsilon=0$ (thick solid line) and $\epsilon=1$ (dot-dashed line). In all plots we considered the ratio of virial to core radius to be $p_{o}=10$ today. In Fig. (1 $\mathrm{b}$ ) we show the effect of varying the core radius for a fixed virial radius today, and no IC gas evolution $(\epsilon=0)$. We considered $p_{o}=7,10,15$. Notice that the amplitude is largest for $\epsilon=-1$ and $p_{o}=7$ since those models lead to larger gas fractions. As expected from our discussion in Sec. 2, all power spectra have a similar bell shape in all cases. The coherence scales are around $l=1000-2000$. Differences in shape are less important than in amplitude. Also, notice that the dependence is stronger on the core radius than on the IC gas evolution. If our cluster model is correct, measuring the anisotropy induced by clusters of galaxies an estimate of the average core radius of the cluster population could be obtained which will only depend marginally on the IC gas evolution.

In Fig. (2a) we show the power spectrum of temperature anisotropies for different mass cut-off: $M_{l}=$ $10^{13}, 5 \cdot 10^{13}, 10^{14} \mathrm{M}_{\odot}$. Notice that the amplitude of the power spectrum is remarkably unsensitive to the change in the lower limit, the reason being that the integrand of eq. [11] is peaked around $2 \cdot 10^{14} \mathrm{M}_{\odot}$ and falls there of, as indicated by Fig. (2b). In this figure, the left solid line displays $d \bar{y} / d M$, the dashed line represents $d P(l) / d M$ for $l=100$ while $l=1000$ is represented by the right solid line. As a result, the bulk of the contribution to the radiation power spectrum comes from clusters of mass close to $10^{14} \mathrm{M}_{\odot}$. So, even though the entropy driven model of clusters we used has not been checked for cluster masses below $10^{14} \mathrm{M}_{\odot}$, the CMB temperature fluctuations shown in Fig. (11) are reasonable accurate. This is not the case for the mean Comptonization parameter $\bar{y}$ which depends strongly on the lower mass cut-off as the contribution of clusters of small mass dominate the integral, as indicated by Fig. (2b). 
In Fig. (3a) we plot the mean Comptonization parameter as a function of the IC gas evolution. We varied the lower mass limit of the integral in eq. [6]. From top to bottom, the thin solid line corresponds to a lower mass limit of $M_{l}=10^{13} \mathrm{M}_{\odot}$, the dash-dotted line to $5 \cdot 10^{13} \mathrm{M}_{\odot}$, and the thick solid line to $10^{14} \mathrm{M}_{\odot}$, all three with $p_{o}=10$. The upper and lower dashed lines correspond to $p_{o}=7,15$, respectively. In the latter two cases the lower mass limit was $10^{14} \mathrm{M}_{\odot}$.

In analogy to the relation eq. [1] we introduce a new variable $\eta$ defined as

$$
\eta \equiv \frac{\sqrt{C(0)}}{\bar{y}}
$$

In Fig. ( $3 \mathrm{~b})$ we plot the behaviour of $\eta$ for the most extreme parameters of the cluster model used. To calculate the correlation function we used SuZie window function. Again, we assumed $g(x)=1$. Lines correspond to the same models as in Fig. (3 a). The curves corresponding to different lower limits on the mass integral are represented twice. We have ploted $\eta$ (lower thin solid and dot dashed lines) and $\eta \sqrt{\bar{n} / n_{c l}}$ (upper thin and dashed-dotted lines). This figure demonstrates that while $C(0)$ and $\bar{y}$ depend strongly on the evolution of the IC gas and the size of the core radius, $\eta$ varies by less than $20 \%$. The largest dependence of $\eta$ comes from varying the present number density of clusters. In the Press-Schechter theory, decreasing the lower mass limit increases $\bar{n}$, the number density of clusters above a given mass scale. In our case, the lower mass limits $M_{l}=10^{13}, 5 \cdot 10^{13}, 10^{14} \mathrm{M}_{\odot}$ correspond to $1.5 \cdot 10^{-3}, 6 \cdot 10^{-4}$ and $2.5 \cdot 10^{-4}$ clusters per $h^{-3} \mathrm{Mpc}^{3}$, respectively. If $C(0)$ and $\bar{y}$ instead of being normalized to $\sigma_{8}$ were normalized to a fixed number of clusters today, temperature correlation and mean distortion would be rescaled as: $C(0) \cdot n_{c l} / \bar{n}$ and $\bar{y} \cdot n_{c l} / \bar{n}$, where $n_{c l}$ is the number density of clusters that produce a measurable effect on the CMB. Then $\eta$ will scale as $\sqrt{\bar{n} / n_{c l}}$. Going back to Fig. (3b) the reader can check that this rescaling renders the solid thin and the dot-dashed lines in the range of $\eta \simeq 2.0$.

Let us remark that the value of $\eta$ is different for different experiments. For Suzie $\eta \simeq 2.0$ and for an experiment with infinite angular resolution $\eta \simeq 2.5$ (see eq. [13]). However, a tight relation between distortion and anisotropy exists, independently of the experiment considered. This tight relation is the consequence of the unsensitivity of the power spectrum with the lower limit of the mass integral.

The upper limits on CMB temperature anisotropies obtained by SuZie (Church et al. 1997) on angular scales of $l \simeq 2000$ can be used to estimate upper limits on the mean Comptonization parameter that are stronger than those set by COBE/FIRAS. Let us assume that that temperature anisotropies on scales measured by SuZie are dominated by clusters, a very conservative assumption for the argument that follows. Using the results summarized in Fig. (3b), we can impose an upper limit on $\bar{y}$ from SuZie upper limit (in absolute value) $\sqrt{C(0)} \leq 2.1 \times 10^{-5}$ at the $95 \%$ confidence level. At $142 \mathrm{GHz}$, SuZie operating frequency, $g(x) \simeq-1$. If we assume that only clusters with masses larger than $10^{14} \mathrm{M}_{\odot}$ contribute to the $\mathrm{CMB}$ distortion, we have $\eta \geq 2.0$. Therefore, $\bar{y} \leq 1.0 \times 10^{-5}$, a contraint stronger than the upper limit obtained by Mather et al. (1994) at the same confidence level. Let us remark that the SuZie upper limits quoted above were obtained assuming Gaussian statistics, while in our analysis we assumed that clusters were Poisson distributed on the sky. A firm upper bound can not be obtained wihtout reanalyzing the SuZie data.

The mean density of clusters can be estimated with independent measurements of distortion and temperature anisotropy. If the contribution of clusters to the temperature fluctuations of the CMB is measured by, say, SuZie and the blackbody distortion is also determined, comparison with Fig (3b) would allow a direct estimate of the number density of clusters with IC gas hot enough to produce a significant contribution to the SZ effect. Comparison of the mean number density of SZ clusters with that of X-ray clusters would lead to a better understanding of the IC gas evolution history.

\section{Conclusions.}

In this paper we have shown that the shape of the power spectrum of temperature anisotropies induced by the hot IC gas is almost independent of model parameters. However, the amplitude does depend on how the mean population of clusters is modeled. As shown by Tegmark (1998) the shape of the power spectrum is important for extracting the foreground contribution on experiments measuring the same region of the sky at different frequencies and angular scales. One could expect the amplitude to be obtained from measurements of temperature anisotropies on experiments like SuZie (Holzapfel et al. 1997), Ryle (Saunders 1997), VSA (Jones 1996) or the upcom- 
ming MAP and Planck satellite missions. This will set strong constraints on the average properties of the cluster population.

We showed that a relation between the temperature anisotropy and the mean Comptonization parameter exists on the average, independent of the cluster model and IC gas evolution. The influence $p_{o}$ shown in Figure 1b and 3a cancels out in the ratio $\eta$. In a first approximation, this ratio only varies with the number density of clusters contributing to SZ effect. This conclusion relies on the assumption that the cluster population as a whole is well described by the scaling relations of eq. ([15]-[17]). This hypothesis, known as Weak Self-Similarity Principle (Bower 1997) do not imply these relations should be accurate for single cluster. For example, asphericity is important when computing the effect of a single cluster on the $\mathrm{CMB}$, but it should average out when considering the effect of the whole cluster population. But, if electron clumpiness or temperature gradients are shown to be common in clusters, this would limit the validity of our results. We do not know how to quantify these effects at the present and for this reason we did not include them into our analysis. Also, let us remark that the influence of different cosmological models through $d V / d z$ has not been addressed in this paper.

To conclude, if our cluster model is applicable to the average cluster population and the number density of SZ clusters is known, then data on temperature anisotropies can be used to determine the distortion of the CMB. On the other hand two independent measurements of temperature anisotropy and distortion would provide an estimate of the number density of Sunyaev-Zel'dovich clusters. Comparison with the number density of X-ray clusters will help to understand cluster formation and evolution.

Acknowledgments. We thank the referee for his careful reading of the manuscript that helped to improve the paper substantially. This research was supported by Spanish German Integrated Actions HA 97/39 . FAB would like to acknowledge the support of the Junta de Castilla y León, grant SA40/97.

\section{REFERENCES}

Atrio-Barandela, F., Gottlöber \& S., Mücket, J. 1997, ApJ, 482, 1

Bartlett, J.G., Silk, J. 1994, ApJ, 423, 12

Bower, R.G. 1997, MNRAS, 288, 355
Colafrancesco, S., Mazzotta, P., Rephaeli, Y. \& Vittorio, N. 1994, ApJ, 433, 454

Colafrancesco, S., Mazzotta, P., Rephaeli, Y. \& Vittorio, N. 1997, ApJ, 479, 1

Colafrancesco, S. \& Vittorio, N. 1994, ApJ, 422, 443

Cole, S. \& Kaiser, N. 1989, MNRAS, 233, 637

Church, S. E., Ganga, K.M., Ade, P. A. R., Holzapfel, W. L., Mauskopf, P. D., Wilbanks, T. M. \& Lange, A. E. 1997 ApJ, 484, 523

Holzapfel, W. L. et al. 1997 ApJ, 480, 449

Hobson, M. P., Jones A. W., Lasenby, A. N. \& Bouchet F. R. 1998, preprint astro-ph/9806387

Jones, M. E. 1996, preprint astro-ph/9611212, in: Proceedings of the XXXIst Recontre de Moriond "Microwave Background Anisotropies"

Jones, C. \& Forman, W. 1984, ApJ, 276, 38

Makino, N., Sasaki, S. \& Suto, Y. 1998, ApJ, 497, 555

Markevitch, M., Forman, W.R., Sarazin, C.L. \& Vikhlinin, A. 1997, preprint astro-ph/9711289

Mather, J.C. et al. 1994, ApJ, 420, 439

Mushotzky, R.F. \& Scharf, C.A. 1997, ApJ, 482, L13

Peebles P.J.E. 1993, " Principles of Physical Cosmology". (Princeton University Press: New Jersey).

Press, W.H., \& Schechter, P. 1974, ApJ, 187, 425

Rephaeli, Y. 1995, ARA\&A, 33, 541

Saunders, R. 1996, astro-ph/9611212, in: Proceedings of the XXXIst Recontre de Moriond "Microwave Background Anisotropies"

Sunyaev, R. \& Zel'dovich, Ya.B. 1970, Ap\&SS, 7, 3

Tegmark, M. \& Efstathiou, G. 1996, MNRAS, 281, 1297

Tegmark, M. 1998, ApJ, 502, 1

Tozzi, P. \& Governato, F. 1997. To appear in "The Young Universe", eds. S. D'Odorico, A. Fontana and E. Giallongo, A.S.P Conf. Ser. astro-ph/9711258

This 2-column preprint was prepared with the AAS LATEX macros v3.0. 

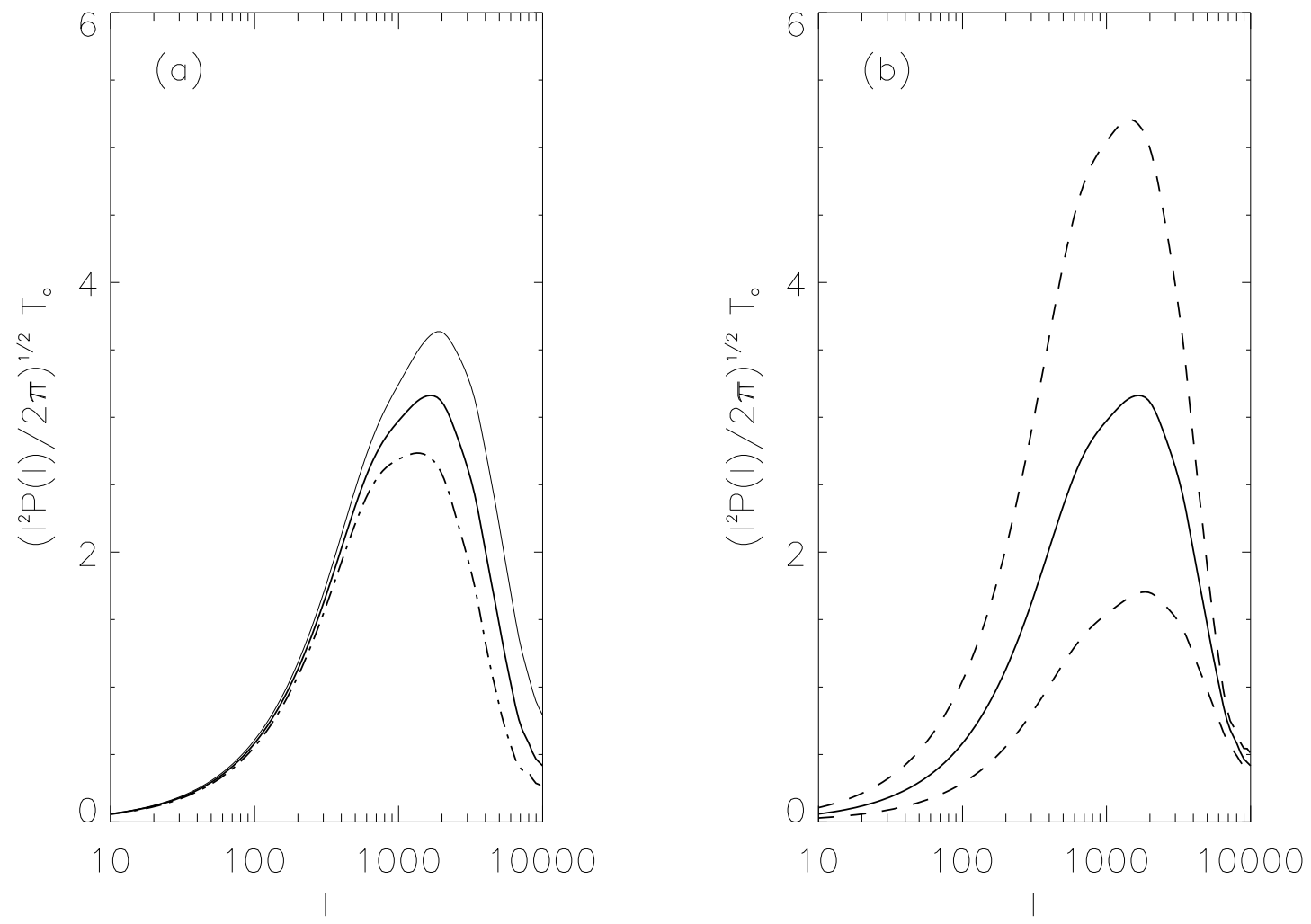

Fig. 1.- Mean CMB temperature offset induced by hot gas in clusters of galaxies expressed in units of $\mu \mathrm{K}$. In (a) plots correspond to different gas evolution histories with identical virial to core radius ratio today $\left(p_{o}=10\right)$ : thin solid line $\epsilon=-1$, thick solid line $\epsilon=0$ and dot-dashed line $\epsilon=1$. In (b) temperature fluctuations for different ratios of virial to core radius today with $\epsilon=0$ are represented: upper dashed line $p_{o}=7$, thick solid line $p_{o}=10$ and lower dashed line $p_{o}=15$. Integration of eq.[11] was performed in the mass range $10^{14} \mathrm{M}_{\odot}<M<2 \cdot 10^{15} \mathrm{M}_{\odot}$. 

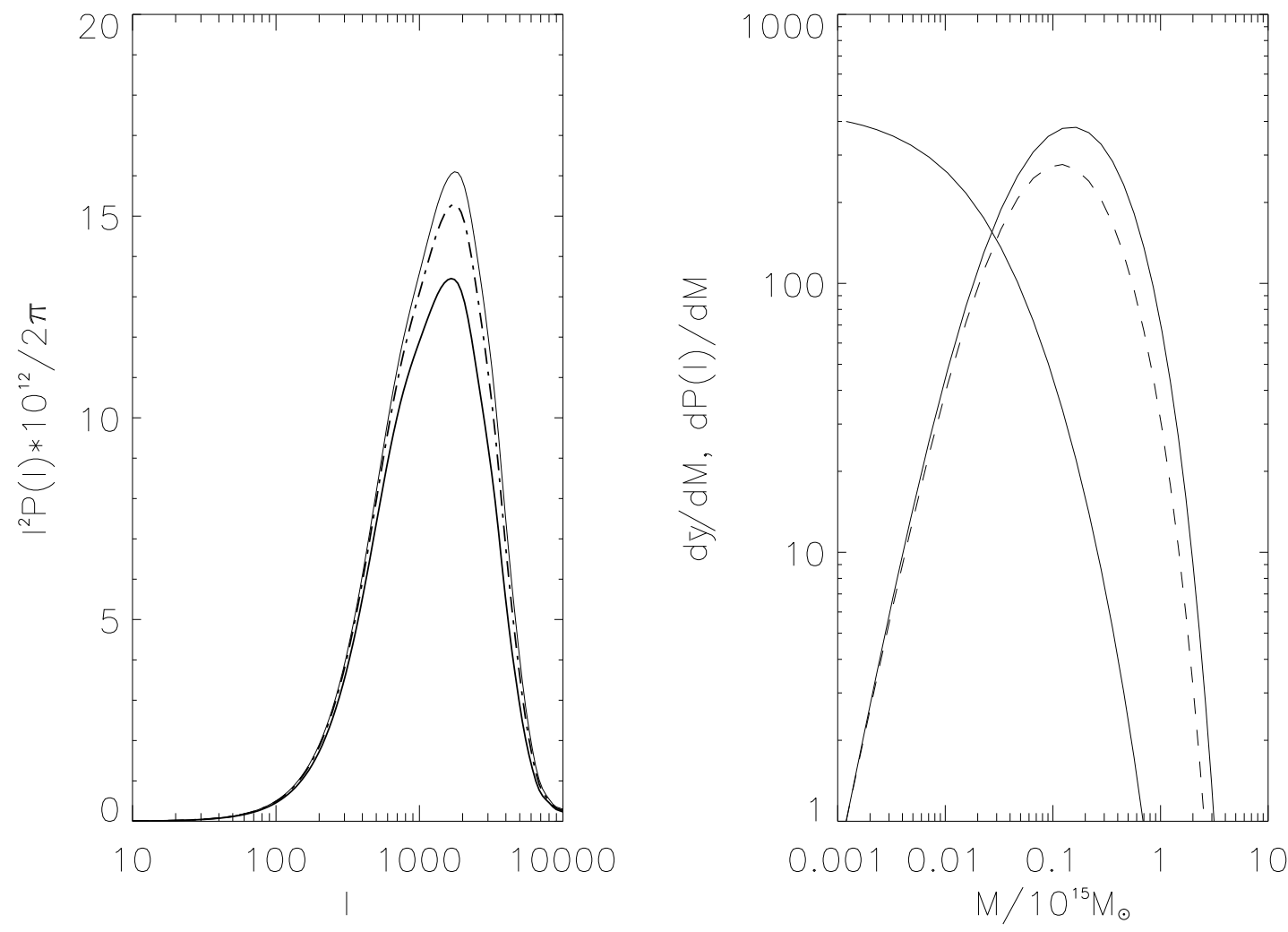

Fig. 2.- (a) Power spectrum of CMB temperature anisotropies induced by hot IC gas. The curves differ in their lower limit in the mass integration of eq. 11]. Solid line correspond to a lower limit of $10^{13} \mathrm{M}_{\odot}$, dot-dashed line to $5 \cdot 10^{13} \mathrm{M}_{\odot}$ and thick solid line to $10^{14} \mathrm{M}_{\odot}$. The mass upper limit was the same as those of Fig. [1]. (b) Left solid line: $d \bar{y} / d M$. Dashed line $d P(l) / d M$ for $l=100$ and right solid line for $l=1000$. The scale on the $\mathrm{y}$-axis is arbitrary and the $d \bar{y} / d M$ curve has been rescaled to fit in the frame. 

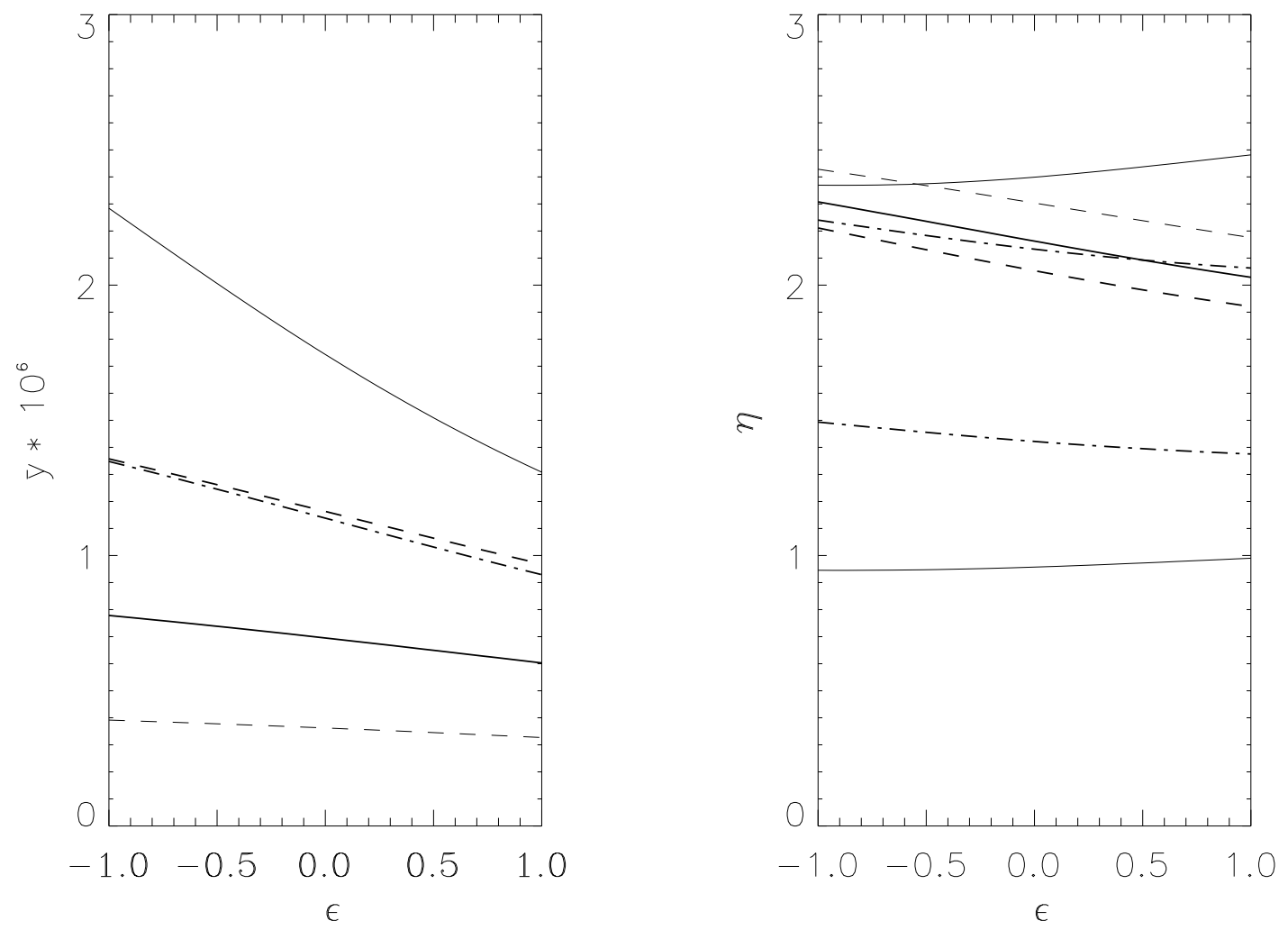

Fig. 3.- (a) Mean Comptonization parameter. Thin solid, dot-dashed and thick solid lines correspond to $p_{o}=10$ and lower limit of mass integration $10^{13}, 5 \cdot 10^{13}$ and $10^{14} \mathrm{M}_{\odot}$, respectively. Upper and lower dashed curves correspond to $p_{o}=7,15$ and $10^{14} \mathrm{M}_{\odot}$ lower limit. (b)Ratio of $\sqrt{C(0)}$ to the mean Comptonization parameter. The two bottom curves correspond to different lower limits on the mass integral (thin solid line $10^{13} \mathrm{M}_{\odot}$ and dot-dashed line $5 \cdot 10^{13} \mathrm{M}_{\odot}$ ). In this two cases the quantity $\eta \sqrt{\bar{n} / n_{c l}}$ was also plotted (upper thin solid and dot-dashed lines). We took the number density of SZ clusters to be $n_{c l}=2.5 \cdot 10^{-4} h^{3} \mathrm{Mpc}^{-3}$. The upper dashed, thick solid and lower dashed lines correspond to $p_{o}=15,10,7$, respectively. 